\title{
Observation d'une toxicité chronique au lithium chez une femme de 54 ans
}

\author{
Jane Kobylianskii MD, Emily Austin MD, Wayne L. Gold MD, Peter E. Wu MD MSc
}

- Citation : CMAJ 2021 August 30;193:E1345-8. doi : 10.1503/cmaj.210725-f

Voir la version anglaise de l'article ici : www.cmaj.ca/lookup/doi/10.1503/cmaj.210725

$\mathbf{U}$

ne femme de 54 ans s'est présentée au service des urgences après avoir vécu 2 jours avec un état mental altéré, de la faiblesse et des mouvements involontaires. Ses antécédents médicaux indiquaient un trouble bipolaire de type 1, diagnostiqué 32 ans auparavant. Elle prenait les médicaments d'ordonnance suivants, administrés 1 fois par jour par voie orale : $900 \mathrm{mg}$ de carbonate de lithium (préparation à libération prolongée), $3 \mathrm{mg}$ de rispéridone, $1 \mathrm{mg}$ de benzatropine, et $0,5 \mathrm{mg}$ de lorazépam (au besoin). Depuis plusieurs années, sa dose de lithium était stable et elle ne présentait pas d'antécédents de surdose aiguë ni d'erreur de dosage. Elle ne consommait pas de médicaments en vente libre ni d'autres substances.

Lors de l'examen, la patiente était afébrile, sa tension artérielle était de 101/54 mm Hg et sa fréquence cardiaque était de 110 battements/ min et régulière. Son état mental était altéré, présentant un score de Glasgow de 10 (E4 V2 M4). Elle avait des tremblements brusques et de l'hyperréflexie accompagnés d'un clonus inductible bilatéral de la cheville. Ses mouvements extraoculaires étaient normaux, sans présence de nystagmus. Aucune anomalie neurologique focale n'était observable. Elle présentait des signes d'hypovolémie intravasculaire avec une basse pression veineuse jugulaire.

Des analyses sanguines ont décelé une glycémie aléatoire de 7,6 (intervalle normal de 3,8 à 7,7 ) $\mathrm{mmol} / \mathrm{L}$; un taux de sodium de 146 (intervalle normal de 135 à 145) $\mathrm{mmol} / \mathrm{L}$; un taux de potassium de 4,0 (intervalle normal de 3,2 à 5,0 ) $\mathrm{mmol} / \mathrm{L}$; un taux de créatine de 115 (intervalle normal de 50 à 98 ) $\mu \mathrm{mol} / \mathrm{L}$ avec un taux de filtration glomérulaire estimé (TFGe) de $46 \mathrm{~mL} /$ $\mathrm{min} / 1,73 \mathrm{~m}^{2}$ (on avait documenté une fonction rénale normale 2 ans auparavant avec un taux de créatine de $89 \mu \mathrm{mol} / \mathrm{L}$ et un TFGe de $\left.64 \mathrm{~mL} / \mathrm{min} / 1,73 \mathrm{~m}^{2}\right)$. Un électrocardiogramme à 12 dérivations affichait un rythme sinusal normal, des inversions précordiales de l'onde $\mathrm{T}$ et un allongement de l'intervalle QTC de 533 ms (figure 1). On n'a observé aucune anomalie intracrânienne aiguë à l'examen tomodensitométrique sans agent de contraste de la tête. Le taux de lithium sérique de la patiente était de $1,50 \mathrm{mmol} / \mathrm{L}$ (intervalle thérapeutique normal de 0,50 à $1,20 \mathrm{mmol} / \mathrm{L}$ ). En raison des symptômes neurologiques de notre patiente, des observations faites sur son électrocardiogramme et de son taux de lithium sérique légèrement élevé, nous avons diagnostiqué une toxicité chronique au lithium.

\section{Points clés}

- Le lithium est un médicament fréquemment utilisé qui possède un indice thérapeutique étroit et qui peut entraîner une toxicité importante qui peut être de nature aiguë, chronique ou aiguë sur fond chronique.

- Les taux sériques de lithium ne sont que l'un des facteurs servant à orienter le diagnostic et la prise en charge; une toxicité chronique cliniquement significative peut se produire chez des patients dont les taux sériques sont peu élevés.

- L'implication précoce du centre régional antipoison est importante pour orienter la prise en charge, y compris la décision d'amorcer l'hémodialyse.

- Un rebond de la présence de lithium peut survenir après l'hémodialyse; on devrait mesurer le taux sérique toutes les 4 heures pour une période d'au moins 12 heures après avoir interrompu l'hémodialyse, s'assurant que la diminution des taux se poursuit.

- Le syndrome de neurotoxicité irréversible imputable au lithium (syndrome of irreversible lithium-effectuated neurotoxicity ou SILENT) est une complication peu fréquente de la toxicité au lithium qui entraîne un dysfonctionnement cérébelleux prédominant et persistant.

Nous avons interrompu son traitement au lithium et lui avons administré une perfusion intraveineuse isotonique. Après 48 heures, son taux de lithium sérique avait diminué à $0,40 \mathrm{mmol} / \mathrm{L}$, mais a ensuite rebondi à $1,30 \mathrm{mmol} / \mathrm{L}$ au troisième jour de l'hospitalisation malgré l'administration en continu de la perfusion intraveineuse. Pendant ce temps, on n'a observé aucune amélioration de l'état mental de la patiente. Nous avons consulté le centre antipoison régional et le service de néphrologie puis nous avons amorcé le traitement d'hémodialyse. Après 8 heures d'hémodialyse à faible efficacité en continu, son niveau de conscience s'est amélioré et son taux de lithium sérique était indétectable. On a surveillé ce taux pendant 48 heures et il est demeuré indétectable. Son rétablissement neurologique s'est bien déroulé lors de son séjour à l'hôpital. Une semaine après son hospitalisation, après l'arrêt de sa perfusion intraveineuse, nous avons observé une hypernatrémie persistante, une polyurie avec une diurèse de $3 \mathrm{~L} / \mathrm{j}$ et une urine diluée 
dont l'osmolalité était de $102 \mathrm{mmol} / \mathrm{kg}$. Nous lui avons administré de la desmopressine sans résultat. Nous avons diagnostiqué un diabète insipide néphrogénique induit par le lithium; en rétrospective, il s'agissait du facteur de déclenchement probable de sa toxicité en raison de l'hypovolémie intravasculaire qui entraîne une insuffisance rénale aiguë et l'incapacité d'excréter le lithium. Nous lui avons prescrit $10 \mathrm{mg}$ d'amiloride, administré par voie orale 1 fois par jour, afin de traiter son diabète insipide néphrogénique, ce qui a normalisé son osmolalité sérique, sa polyurie et son taux de sodium. Nous avons consulté le service de psychiatrie et avons amorcé un traitement à l'acide valproïque afin de prendre en charge son trouble bipolaire de type 1.

\section{Discussion}

Aux États-Unis, en 2019, on a documenté 7085 expositions toxiques au lithium ${ }^{1}$, ce qui constitue un nombre important d'expositions qui peuvent avoir entraîné des résultats cliniques de gravité modérée, à tout le moins ${ }^{1}$. De ceux-ci, $22 \%$ ont provoqué des conséquences cliniques graves, dont 4 décès ${ }^{1}$. L'indice thérapeutique du lithium est étroit et ce composé possède un vaste éventail de toxicités. Il s'agit d'une thérapie efficace et prescrite à grande échelle contre les troubles bipolaires qui est aussi employée en dehors des indications pour d'autres troubles psychiatriques ${ }^{2}$. Elle est disponible sous forme de préparations à libération immédiate et à libération prolongée ${ }^{2}$. L'intervalle

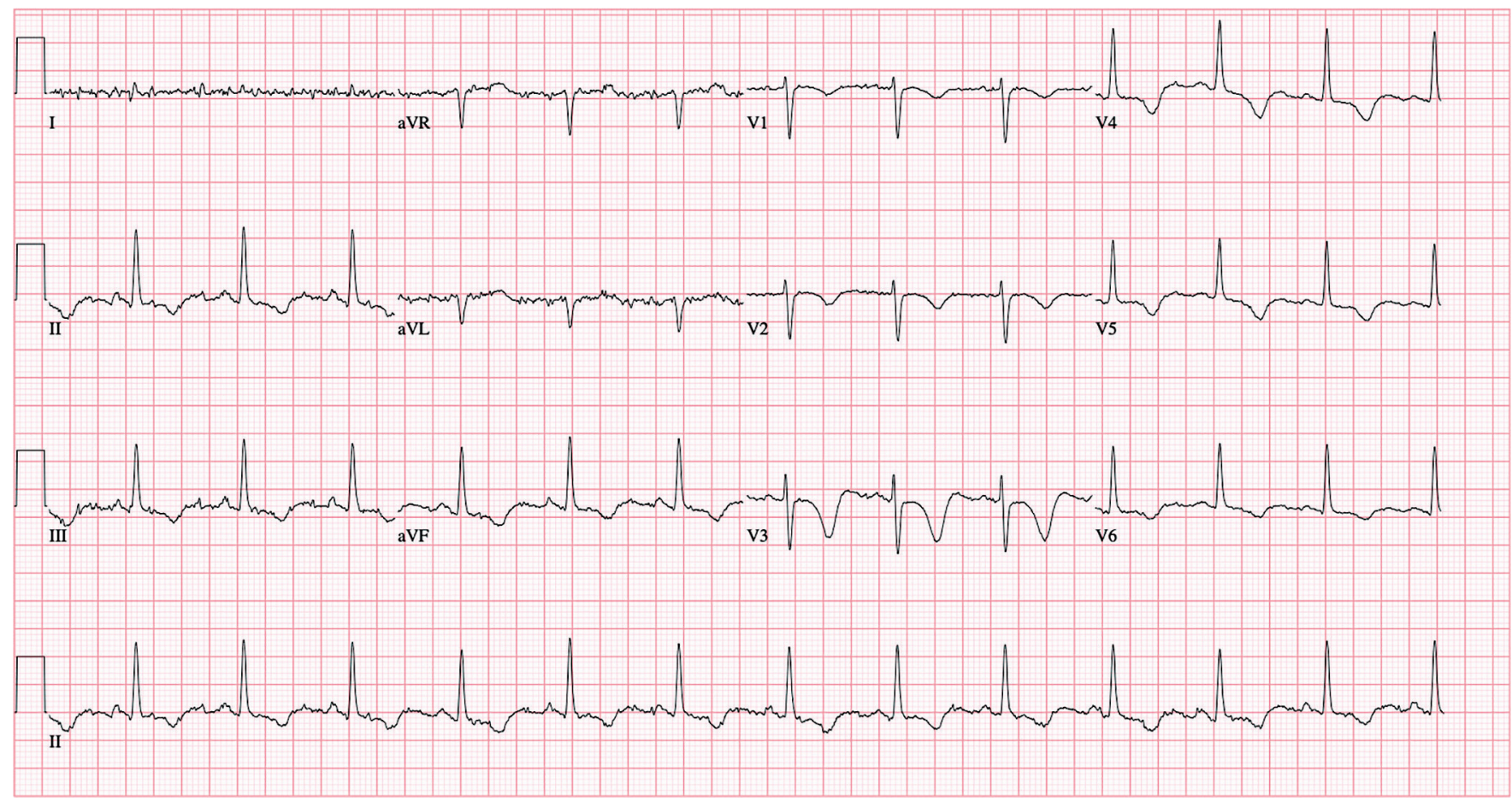

Figure 1 : Électrocardiogramme d'une femme de 54 ans présentant une toxicité chronique au lithium, montrant un rythme sinusal normal, des inversions précordiales de l'onde T et un allongement de l'intervalle QTc de $533 \mathrm{~ms}$.

Tableau 1 : Caractéristiques cliniques de la toxicité au lithium

\section{Schéma}

d'exposition

Aiguë

Chronique

Aiguë sur fond chronique

Ensemble des expositions

\section{Caractéristiques cliniques}

- Gastro-intestinales : nausées, vomissements, diarrhées

- Neurologiques : légères et retardées

- Neurologiques : tremblements, hyperréflexie, myoclonus, faiblesse, ataxie, rigidité, dysarthrie, nystagmus, état de conscience altéré, convulsions

- Rénales : diabète insipide néphrogénique, néphropathie tubulo-interstitielle chronique

- Endocrines : hypothyroïdie, hyperthyroïdie, hyperparathyroïdie, hypercalcémie

- Syndrome de neurotoxicité irréversible imputable au lithium (SILENT)

- Présente les caractéristiques des toxicités aiguë et chronique

- Manifestations gastro-intestinales et neurologiques marquées

- Cardiaque : changements à l'électrocardiogramme (inversions de l'onde T, allongement de l'intervalle QT, affaissement du segment ST, dysfonctionnement du nœud sinoauriculaire, bradycardie), rare manifestation de dysrythmies 
thérapeutique recommandé varie selon l'âge du patient en raison des modifications de la fonction rénale liées à l'âge et à la prévalence accrue de la polypharmacie et des interactions médicamenteuses $^{2}$. Le lithium est un cation monovalent inorganique dont le faible poids moléculaire correspond à $7 \mathrm{Da}^{3}$. Il s'absorbe rapidement, n'est pas lié à une protéine, présente un faible volume de distribution (entre 0,6 et $0,9 \mathrm{~L} / \mathrm{kg}$ ) et est librement excrété par les reins sans être transformé ${ }^{3}$.

Les manifestations cliniques de la toxicité au lithium dépendent de la nature de l'exposition : aiguë, chronique ou aiguë sur fond chronique (tableau 1).

\section{Toxicité aiguë au lithium}

La toxicité aiguë au lithium fait référence à l'ingestion de lithium par un patient qui n'y a jamais été exposé, sans concentration tissulaire préalable $e^{4}$. Cela se produit généralement dans le contexte d'une surdose $e^{4}$. Dans le cas d'une toxicité aiguë, les symptômes gastro-intestinaux sont importants, dont des nausées, des vomissements et de la diarrhée ${ }^{3}$. Bien que des symptômes neurologiques peuvent se manifester, ils sont souvent légers ou retardés en raison de la lente distribution au cerveau qui peut prendre jusqu'à 24 heures après l'ingestion ${ }^{3}$. On peut observer des anomalies électrocardiographiques, dont une inversion de l'onde $\mathrm{T}$, un allongement de l'intervalle QT, un affaissement du segment ST et de la bradycardie, bien qu'une toxicité cardiaque importante soit peu fréquente ${ }^{3}$. On a aussi associé la toxicité aiguë au lithium au syndrome sérotoninergique ${ }^{5}$.

\section{Toxicité chronique au lithium}

La toxicité chronique se manifeste chez les patients qui ont recours au lithium sur une longue période, qui ont accumulé une concentration tissulaire et qui ont ensuite développé un problème de santé qui altère son élimination rénale en diminuant la filtration glomérulaire ou en accroissant sa réabsorption au niveau du tubule proximal ${ }^{4}$. Les facteurs de risque fréquents comprennent l'hypovolémie et l'utilisation concomitante de médicaments néphotoxiques ${ }^{4}$. Dans le cas de la toxicité chronique, les manifestations sont principalement d'ordre neurologique, dont des tremblements, de l'hyperréflexie, un myoclonus, de l'ataxie et du nystagmus $^{3}$. Un spectre d'altérations de l'état mental peut se produire, allant de l'agitation et de la confusion au coma et aux convulsions ${ }^{3}$.
Les complications rénales comprennent une néphropathie tubulointerstitielle chronique et un diabète insipide néphrogénique ${ }^{6}$. Ce dernier est fréquent parmi les patients qui suivent une thérapie chronique au lithium et qui peut mener à une hypovolémie intravasculaire et à une insuffisance rénale aiguë, prédisposant les patients à l'accumulation de lithium, comme nous l'avons soupçonné pour notre patiente ${ }^{6}$. Des anomalies électrocardiographiques peuvent aussi être observées dans les cas de toxicité chronique ${ }^{3}$.

\section{Toxicité aiguë sur fond chronique au lithium}

La toxicité aiguë sur fond chronique fait référence à l'ingestion de lithium au-delà de la posologie de maintien d'un patient ${ }^{4}$. Dans le cas d'une toxicité aiguë sur fond chronique, les patients peuvent présenter un mélange de caractéristiques cliniques, dont des symptômes neurologiques et gastro-intestinaux importants ${ }^{3}$.

\section{Prise en charge de la toxicité au lithium}

Le taux de lithium sérique ne concorde pas de façon fiable avec la gravité clinique de la toxicité ${ }^{3}$. Notre patiente souffrait d'une grave toxicité au lithium tout en présentant un taux sérique peu élevé. On observe souvent ce profil dans les cas de toxicité chronique au lithium en raison de l'accumulation préexistante du composé dans le cerveau et les tissus périphériques ${ }^{3}$. Une affection légère et aiguë peut entraîner des modifications à la fonction rénale qui peuvent créer les conditions pour une toxicité chronique chez les patients traités au lithium ${ }^{4}$. Par conséquent, peu importe le taux de lithium sérique, les cliniciens devraient systématiquement soupçonner une toxicité chronique au lithium dans le cas de tout changement clinique chez un patient suivant une thérapie au lithium sur une longue période.

La prise en charge de la toxicité au lithium comprend des soins de soutien, protégeant spécifiquement les voies respiratoires si le patient présente un état mental altéré, une interruption de l'exposition au lithium et un rehaussement de l'élimination rénale $e^{4}$. Une intervention précoce du centre antipoison local est importante pour orienter la prise en charge. II n'existe pas d'antidote à la toxicité au lithium. Bien que le charbon activé ne puisse jouer aucun rôle, puisqu'il n'absorbe pas le lithium, les cliniciens pourraient considérer une irrigation intestinale totale, particulièrement chez les patients qui ont ingéré des préparations à libération prolongée, pourvu qu'il n'existe pas de contre-indications ${ }^{3}$.

Tableau 2 : Directives du Groupe de travail sur les traitements extracorporels dans les cas d'empoisonnement pour I'hémodialyse dans le cas de toxicité au lithium ${ }^{8}$

\begin{tabular}{|c|c|c|c|}
\hline Directives & $\begin{array}{l}\text { Force des } \\
\text { recommandations }\end{array}$ & Taux de lithium sérique & Caractéristiques cliniques \\
\hline \multirow[t]{2}{*}{$\begin{array}{l}\text { Amorce de } \\
\text { l'hémodialyse }\end{array}$} & Recommandée & $>4,0 \mathrm{mmol} / \mathrm{L}$ avec TFG anormale & $\begin{array}{l}\text { État de conscience diminué, convulsions ou dysrythmies } \\
\text { potentiellement mortelles }\end{array}$ \\
\hline & Suggérée & $>5,0 \mathrm{mmol} / \mathrm{L}$ & $\begin{array}{l}\text { État confus ou taux de lithium dont on ne s'attend pas qu'il chute } \\
\text { sous } 1,0 \mathrm{mmol} / \mathrm{L} \text { au cours des } 36 \text { prochaines heures avec une prise } \\
\text { en charge optimale }\end{array}$ \\
\hline $\begin{array}{l}\text { Arrêt de } \\
\text { l'hémodialyse }\end{array}$ & Recommandée & $<1,0 \mathrm{mmol} / \mathrm{L}$ & $\begin{array}{l}\text { Amélioration apparente de l'état clinique (un minimum de } 6 \mathrm{~h} \\
\text { d'hémodialyse si le taux de lithium sérique n'est pas disponible) }\end{array}$ \\
\hline
\end{tabular}


L'élément le plus important de la prise en charge est l'administration d'une perfusion intraveineuse isotonique afin d'améliorer le taux de filtration glomérulaire (TFG) et faciliter l'élimination rénale ${ }^{4}$. Si cela échoue, un traitement extracorporel faisant appel à l'hémodialyse est hautement efficace pour retirer le lithium en raison de ses propriétés pharmacocinétiques, dont son faible poids moléculaire et la petite taille de son volume de distribution ${ }^{4}$. Bien qu'on ait largement exploré la chronicité de l'utilisation de l'hémodialyse chez les patients atteints de toxicité au lithium, les recommandations spécifiques à son utilisation varient grandement. Cela est possiblement le reflet des lacunes en matière de données probantes et de l'hétérogénéité du tableau clinique des patients atteints de toxicité au lithium ${ }^{4,7}$. En général, on prend en considération l'état clinique du patient, sa fonction rénale et son taux de lithium sérique lorsqu'on prend la décision d'offrir l'hémodialyse. En 2015, le Groupe de travail sur les traitements extracorporels dans les cas d'empoisonnement a proposé un ensemble de directives pour l'hémodialyse dans les cas de toxicité au lithium, reconnaissant les limites des données probantes disponibles (tableau 2$)^{8}$.

Le rebond de lithium fait référence à un phénomène où le taux de lithium sérique augmente après avoir initialement diminué ${ }^{8}$. Cela se produit le plus souvent après l'arrêt d'une hémodialyse intermittente chez les patients traités pour une toxicité chronique au lithium ${ }^{8}$. Le lithium est rapidement éliminé du compartiment sanguin, en raison de son faible volume de distribution; cependant, le transfert transcellulaire et la lente diffusion du lithium à l'extérieur des tissus peuvent entraîner un phénomène de rebond ${ }^{9}$. Ce rebond peut aussi se produire lors de l'ingestion d'une préparation à libération prolongée en raison de son absorption continue ${ }^{9}$. Dans notre cas, nous avons observé le rebond de lithium après le traitement de réanimation liquidienne par intraveineuse uniquement, avant que la patiente ne subisse une hémodialyse. On doit s'assurer que le taux de lithium sérique décroît de façon soutenue ou qu'il est indétectable avant de déterminer si on doit poursuivre le traitement. Pour cette raison, on recommande de mesurer le taux sérique toutes les 4 heures pour une période d'au moins 12 heures après avoir arrêté l'hémodialyse ${ }^{8}$.

Finalement, le dysfonctionnement neurologique prolongé constitue une importante complication de l'empoisonnement au lithium ${ }^{4}$. Le syndrome de neurotoxicité irréversible imputable au lithium (syndrome of irreversible lithium-effectuated neurotoxicity ou SILENT) se caractérise par des changements neurologiques persistant sur une période d'au moins 2 mois malgré le retrait de la thérapie au lithium ${ }^{10}$. Ce syndrome se manifeste largement au niveau du cervelet et peut comprendre des tremblements, un nystagmus et de la dysarthrie, entre autres ${ }^{10}$. Il demeure incertain si une hémodialyse précoce ou agressive prévient le syndrome de neurotoxicité irréversible imputable au lithium et quels sont les principaux facteurs de risque qui prédisposent à ce trouble ${ }^{10}$.

Le lithium est un médicament fréquemment prescrit présentant un étroit indice thérapeutique et qui peut causer une toxicité aiguë, chronique ou aiguë sur fond chronique Le diagnostic de la toxicité au lithium ne devrait pas reposer uniquement sur le taux sérique. Il s'agit principalement d'un traitement de soutien où on porte une attention à l'optimisation de l'élimination rénale, bien que l'hémodialyse puisse être indiquée. Notre patiente a dû subir des traitements d'hémodialyse malgré une réanimation liquidienne vigoureuse. Elle s'est par la suite rétablie et son état s'est maintenu depuis son congé de l'hôpital.

\section{Références}

1. Gummin DD, Mowry JB, Beuhler MC, et al. 2019 annual report of the American Association of Poison Control Centers' National Poison Data System (NPDS): 37th annual report. Clin Toxicol (Phila) 2020;58:1360-541.

2. Yatham LN, Kennedy SH, Parikh SV, et al. Canadian Network for Mood and Anxiety Treatments (CANMAT) and International Society for Bipolar Disorders (ISBD) 2018 guidelines for the management of patients with bipolar disorder. Bipolar Disord 2018;20:97-170.

3. Greller H. Lithium. In: Nelson LS, Howland MA, Lewin NA, editors. Goldfrank's toxicologic emergencies. 11th ed. New York: McGraw-Hill Education; 2019.

4. Baird-Gunning J, Lea-Henry T, Hoegberg LCG, et al. Lithium poisoning. J Intensive Care Med 2017;32:249-63.

5. Boyer EW, Shannon M. The serotonin syndrome. N Engl J Med 2005;352:1112-20.

6. Grünfeld J-P, Rossier BC. Lithium nephrotoxicity revisited. Nat Rev Nephrol 2009; 5:270-6.

7. Lavonas EJ, Buchanan J. Hemodialysis for lithium poisoning. Cochrane Database Syst Rev 2015;(9):CD007951.

8. Decker BS, Goldfarb DS, Dargan PI, et al. Extracorporeal treatment for lithium poisoning: systematic review and recommendations from the EXTRIP workgroup. Clin J Am Soc Nephrol 2015;10:875-87.

9. Timmer RT, Sands JM. Lithium intoxication. J Am Soc Nephrol 1999;10:666-74.

10. Adityanjee, Munshi KR, Thampy A. The syndrome of irreversible lithiumeffectuated neurotoxicity. Clin Neuropharmacol 2005;28:38-49.

\section{Intérêts concurrents : Aucun déclaré.}

Cet article a été révisé par des pairs.

Les auteurs ont obtenu le consentement de la patiente.

Affiliations : Département de médecine (Kobylianskii, Austin, Gold, Wu), Université de Toronto; Centre antipoison de l'Ontario (Austin), Hôpital pour enfants malades; Division de pharmacologie clinique et de toxicologie (Austin, Wu), Université de Toronto; Division de médecine interne générale (Gold, Wu), Réseau universitaire de santé/ Système de santé Sinaï, Toronto, Ont.

Collaborateurs : Jane Kobylianskii a contribué à l'élaboration et à la conception du travail, elle a recueilli les données de la patiente et elle a produit la première ébauche du manuscrit. Emily Austin et Wayne Gold ont contribué à l'interprétation des données et ils ont révisé de façon critique le contenu intellectuel important du manuscrit. Peter Wu a contribué à l'élaboration et à la conception du travail ainsi qu'à l'interprétation des données et il a révisé de façon critique le contenu intellectuel important du manuscrit. Tous les auteurs ont donné leur approbation finale pour la version destinée à être publiée et endossent l'entière responsabilité de tous les aspects du travail.

Propriété intellectuelle du contenu : Il s'agit d'un article en libre accès distribué conformément aux modalités de la licence Creative Commons Attribution (CC BY-NC-ND 4.0), qui permet l'utilisation, la diffusion et la reproduction de tout médium à la condition que la publication originale soit adéquatement citée, que l'utilisation se fasse à des fins non commerciales (c.-à-d., recherche ou éducation) et qu'aucune modification ni adaptation n'y soit apportée. Voir : https://creativecommons.org/licenses/by-nc-nd/4.0/deed.fr.

Correspondance : Jane Kobylianskii, Jane.kobylianskii@mail.utoronto.ca 